\title{
Shakedown assessment of multilayered thermal barrier systems considering interface imperfections
}

\author{
Xiaotao Zheng ${ }^{1}$, Haofeng Chen ${ }^{2 *}$, Zhiyuan $\mathrm{Ma}^{2}$ \\ 1. Hubei Provincial Key Laboratory of Chemical Equipment Intensification and \\ Intrinsic Safety, Wuhan Institute of Technology, Wuhan 430205, P. R. China \\ 2. Department of Mechanical \& Aerospace Engineering, University of Strathclyde, \\ Glasgow G1 1XJ, UK
}

\begin{abstract}
:
Thermal barrier coatings (TBCs) usually have complex structures and operate in cyclic high temperatures. The cracking phenomenon is usually observed at the interface with microscopic imperfections between the bond and the thermally grown oxide (TGO) layer owing to the cyclic plasticity around the defect interface under the temperature cycling. Shakedown limits of TBCs considering the impacts of interface imperfections are investigated based on the linear matching method. The temperature-dependent yield stress, geometric parameters $h_{o}, R / h_{o}$ and $H / R$ as well as the thermal expansion coefficient ratio $\alpha / \alpha_{\mathrm{r}}$ are discussed in detail. Results present that the thickness of the TGO has no effect and the geometric factor $R / h_{o}$ shows a little influence on shakedown limits of the multilayered systems. However, the geometric parameter $H / R$ and the thermal expansion coefficient ratio $\alpha / \alpha_{\mathrm{r}}$ have a remarkable impact on shakedown limits. This indicates that the depth of the defect influences shakedown limits greatly, whereas the transition radius at the corner of the imperfection shows slight effect. This is obviously different with the common phenomenon that the transition radius usually has an important influence on shakedown limits. Based on the calculated data, the assessment approach of shakedown limits for TBCs considering various factors mentioned above is proposed. It is of great interest that the simulated effective plastic strain above the shakedown limit load occurs at the defect interface between the TGO and the bond layer, which is

${ }^{1}$ Author to whom correspondence should be addressed;

Electronic mail: haofeng.chen@strath.ac.uk
\end{abstract}


the same crack failure position comparing with the microscopic morphology observation for cracked TBCs.

Keywords: Shakedown; Thermal barrier coatings; Imperfections; Temperature-dependent yield stress; Linear Matching method

\section{Introduction}

Until now, hundreds of different types of coatings are developed to protect various engineering structures from wear, corrosion, erosion, thermal failure and so on. In these coatings, TBCs usually have the most complex structure and operate in very high temperature condition. TBCs are usually used to insulate turbine and combustor engines from the high-temperature stream, and enhance the service life and energy efficiency of these components [1]. Generally, TBCs are typical multilayered systems and are mainly composed by four parts: TBC itself, the superalloy substrate, the bond coat between the TBC and the substrate, and TGO that produces between the bond layer and the TBC. The TBC is the insulator of the multilayered systems, the bond coat is the protection layer from oxidation, and the superalloy substrate is the main structure to resist the external loads. In fact, the TGO is the product of oxidation reaction between the bond layer and the $\mathrm{TBC}$ during the operating process at high temperature. Therefore, extremely large stresses are always produced due to the thermal expansion mismatch between the substrate layer, the bond layer and the TGO layer. In this case, the cracking behavior is usually observed at the interfaces of these layers, especially at the interface between of the bond layer and the TGO layer with microscopic imperfections caused by the stress concentration effect. It is of more importance that severe ratcheting or accumulated plastic strain will take place in these regions due to cyclic thermal loads during the operation process. It is considered as the main factor for the cracking failure of multilayered systems near the interface imperfections [2, 3], as shown in Fig.1. Karlsson et al. simulated the displacement instabilities of the TGO coat produced by interface imperfections to discuss the cyclic 
plasticity around the defects of the bond layer. Results indicate that the plastic zone depends on the temperature cycling. The work is very helpful for explaining the failure mechanism and establishing the safety assessment approach of TBCs under repeated thermal loads.

Until now, many researches have been performed for the stress distribution of multilayered systems under thermal and mechanical loads. To obtain the elastic stress state of multilayered systems, Hsueh [4] and Zhang [5] proposed a three-variable and a two-variable analytical model, respectively. Sometimes, the local plastic deformation is inevitable due to the thermal mismatch for multilayered structures. $\mathrm{Hu}$ [6] and Zhang [7] developed the closed-form formulations of multilayered systems taking into consideration the linear strain-hardening to solve the stress distribution under the local plastic strain condition. Moreover, creep and relaxation behaviors of multilayered systems take place even at room temperature, but that will be much more significant at relatively high temperature [8]. Chen and Xuan [9, 10], Limarga [11] deduced the time-dependent creep deformation and stress state of multilayered systems considering the elastic-creep condition. Mao [12] developed the theory to obtain the plastic-creep behaviors of multilayered structures subjected to cyclic thermal loads based on the elastic-perfectly plasticity. Additionally, for the sake of predicting the more realistic elastic-plastic-creep behaviors of multilayered systems, Eslami and Mahbadi [13] introduced the Prager kinematic hardening model, and Nakane and Ohno et al. [14, 15] considered the more appropriate kinematic strain-hardening constitutive modes to describe the cyclic stress-strain relationships under elastic-plastic-creep conditions. However, according to the best knowledge of authors, very few investigations on the shakedown boundaries of multilayered systems have been addressed, especially for the TBCs with interface imperfections. It is known that the shakedown limit is an important index to guard against the accumulated plastic deformation or ratcheting effect of structures under cyclic loads $[16,17,18]$. Accordingly, it is still necessary to discuss the shakedown limits of multilayered TBCs with interface imperfections for the safety evaluation. 
Experimental observation in the reference [3]

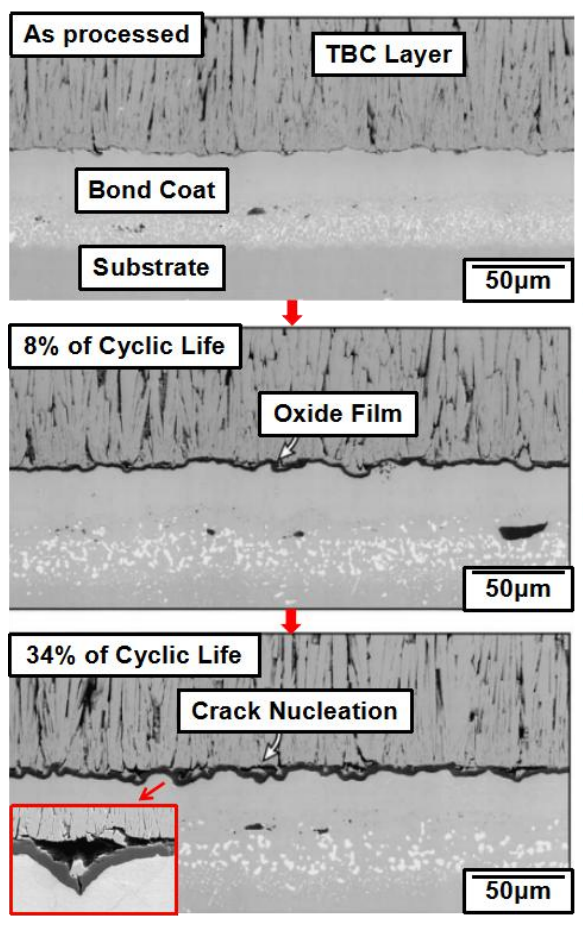

Schematic description

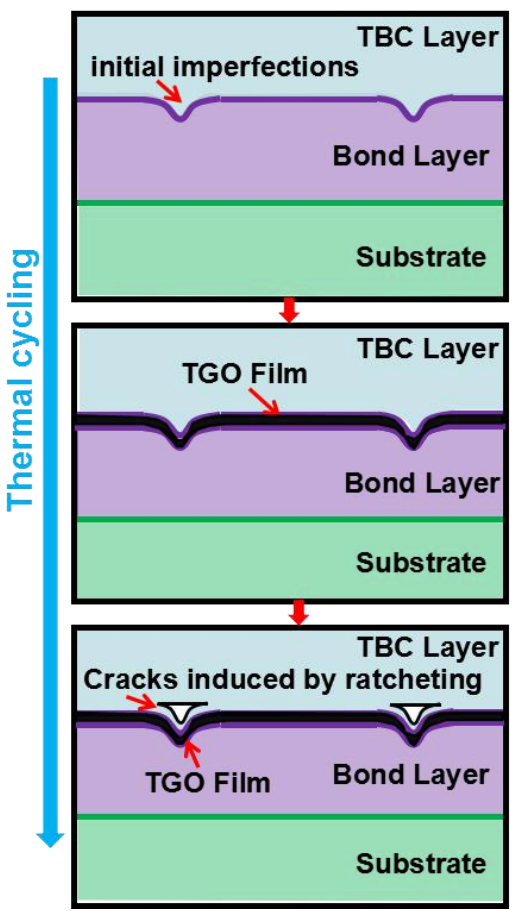

Fig.1 Damage mechanism of TBCs induced by ratcheting deformation

Actually, the ratcheting and shakedown limit assessment is hard to incorporate the design process for engineering applications due to the complexity of classical shakedown theory and geometrical structures. One typical estimation method for complex engineering structures in the existed code EN13445 is to simulate the cyclic elastic-plastic strain response directly by incremental finite element analysis under the given loading conditions, even over 500 cycles [19]. The best advantage of this approach are simple for engineering design and applicable for various complex structures and load histories. However, it is thought to be considerably time consuming. To decrease the numerical expense, a direct cyclic analysis method is proposed and incorporated into the ABAQUS by Nguyen-Tajan et al. to obtain the stabilized feature [20]. Whereas, both approaches mentioned above are only used to estimate the shakedown behavior of engineering structures under a specified load history, such as elastic shakedown, plastic shakedown or ratcheting state, and are not applicable for the prediction of ratcheting and shakedown boundaries. Therefore, a 
large number of simulations should be performed under various load histories to assess the ratcheting and shakedown limits approximately. Generally, the shakedown limit can be calculated directly based on classical static or kinematic theorem. This alternative direct analysis approach is considered to be better than the full step-by-step method mentioned above due to the high efficiency. Recently, the direct analysis approaches based on classical static or kinematic theorem developed rapidly, such as the linear matching method [21, 22], the non-cyclic method [23, 24], the elastic compensation method [25], the generalized local stress strain $\tau$-node method [26] and some other mathematical programming methods [27, 28]. Among this direct analysis approaches, the linear matching method based on the kinematic theory of Koiter [29] has been verified to calculate the precise upper bound shakedown boundaries with relatively high efficiency [30].

In this work, the linear matching method based on the kinematic theory is utilized to analyze shakedown limits of multilayered TBCs with interface imperfections. The influences of key geometric parameters and thermal expansion coefficients are investigated systematically to discuss the shakedown assessment approach of multilayered systems with imperfections for engineering applications. Considering the material performance of the bond layer is typical temperature-dependent, which impacts the shakedown limit of structures obviously, the temperature-dependent yield stress of the bond layer is considered to discuss the shakedown boundaries of TBCs subjected to cyclic temperature loads.

\section{Basic theory for shakedown limit analysis by the linear matching method}

Assuming the material of the bond layer is elastic-perfectly plastic and meets the von Mises yield condition, the TGO layer and the substrate is totally linear-elastic. Taking account of the cyclic high temperature cases of multilayered systems, the temperature-dependent yield stress of the bond layer, $\sigma_{y}^{b c}(T)$, is considered. To achieve the temperature-dependent yield stress changing with the current temperature $T$, the yield stress $\sigma_{y}^{b c}(T)$ is updated during the iterative calculation process. 
If a cyclic temperature field $\lambda \theta\left(x_{i}, t\right)$ for the whole inner body and the surface loads $\lambda P_{i}\left(x_{i}, t\right)$ acting on the specified area $S_{T}$ are applied. Where, $\lambda$ represents a load factor, which is used to calculate all of the load histories. On the surface without surface loads, $S_{r}$, the displacement equals zero. Moreover, if the elastic thermal stress histories $\tilde{\sigma}_{i j}^{\theta}$ under temperature loads $\theta\left(x_{i}, t\right)$ and the elastic mechanical stress histories $\widetilde{\sigma}_{i j}^{p}$ under surface loads $P_{i}\left(x_{i}, t\right)$ can be solved, the linear elastic stress histories of the structure under combined thermal-mechanical loads can be calculated by the superposition principle:

$$
\lambda \widetilde{\sigma}_{i j}=\lambda \widetilde{\sigma}_{i j}^{\theta}+\lambda \widetilde{\sigma}_{i j}^{P}
$$

Under cyclic temperature loads, the stress histories during a typical cycle $0 \leq t \leq \Delta t$ is

$$
\sigma_{i j}\left(x_{i}, t\right)=\lambda \widetilde{\sigma}_{i j}\left(x_{i}, t\right)+\bar{\sigma}_{i j}\left(x_{i}\right)+\widetilde{\sigma}_{i j}^{r}\left(x_{i}, t\right)
$$

Here, $\tilde{\sigma}_{i j}^{r}\left(x_{i}, t\right)$ represents the residual stress components changing with time, and $\bar{\sigma}_{i j}^{r}\left(x_{i}\right)$ is a constant residual stress in equilibrium with external surface loads on $S_{T}$. It should be noted that $\tilde{\sigma}_{i j}^{r}\left(x_{i}, t\right)$ is equal to zero for the shakedown condition. Therefore, the cyclic stress field under the shakedown condition becomes

$$
\sigma_{i j}\left(x_{i}, t\right)=\lambda \widetilde{\sigma}_{i j}\left(x_{i}, t\right)+\bar{\sigma}_{i j}\left(x_{i}\right)
$$

Taking into account the temperature-dependent yield criterion, the relationship between the strain rate $\dot{\varepsilon}_{i j}^{i}$, the shear modulus $G$ and the temperature-dependent yield stress is

$$
\sigma_{y}^{b c}(T)=\frac{3}{2} G \overline{\dot{\varepsilon}}^{i}
$$

Where, $\overline{\dot{\varepsilon}}^{i}$ is the equivalent strain rate, and $\overline{\dot{\varepsilon}}^{i}=\sqrt{\frac{2}{3} \dot{\varepsilon}_{i j}^{i} \dot{\varepsilon}_{i j}^{i}}$.

For a given shear modulus $G$, the incompressible relationship at each instant is proposed under a constant residual stress field $\bar{\sigma}_{i j}^{f}$ in a cycle, as the following 


$$
\left(\dot{\varepsilon}_{i j}^{f}\right)^{\prime}=\frac{1}{G(t)}\left(\lambda_{u b}^{i} \widetilde{\sigma}_{i j}+\bar{\sigma}_{i j}^{f}\right)^{\prime}, \quad \dot{\varepsilon}_{k k}^{f}=0
$$

Where, the load factor $\lambda_{u b}^{i}$ is the upper bound for $\dot{\varepsilon}_{i j}^{i}$. The constant residual stress field $\bar{\sigma}_{i j}^{f}$ can be calculated by integrating Eq.(5) over the whole cycle. Accordingly, the increment of plastic strain over the cycle $\Delta \varepsilon_{i j}^{f}$ and $\bar{\sigma}_{i j}^{f}$ shows the following linear relationship:

$$
\Delta \dot{\varepsilon}_{i j}^{\prime f}=\frac{1}{\bar{G}}\left(\sigma_{i j}^{\prime i n}+\bar{\sigma}_{i j}^{\prime f}\right), \Delta \varepsilon_{k k}^{f}=0
$$

Here, $\sigma_{i j}^{\text {in }}=\bar{G}\left\{\int_{0}^{\Delta t} \frac{1}{G(t)} \lambda_{U B}^{i} \widetilde{\sigma}_{i j}^{\prime}(t) d t\right\}$ and $\frac{1}{\bar{G}}=\int_{0}^{\Delta t} \frac{1}{G(t)} d t$

Therefore, the upper bound on the shakedown limit is obtained by

$$
\lambda_{U B}^{f}=\frac{\int_{V} \int_{0}^{\Delta t} \sigma_{y}^{b c}(T) \cdot \overline{\dot{\varepsilon}} \cdot \dot{\varepsilon}_{i j}^{f} d t d V}{\int_{V}^{\Delta t} \int_{0}^{\Delta t} \widetilde{\sigma}_{i j} \cdot \dot{\varepsilon}_{i j}^{f} d t d V}
$$

The minimum upper bound can be achieved by repeating the above process. To analyze the shakedown boundaries of multilayered TBCs with compex imperfections easily, the above numerical algorithm is implemented into the general commercial finite element software ABAQUS [31] by UMAT user subroutine. The iterative algorithm assesses a varying shear modulus $G$ firstly by matching the stress produced by the linear model proposed above and the temperature-dependent yield criterion at the strain rate $\dot{\varepsilon}_{i j}^{i}$ is calculated by the previous iterative step.

\section{Finite element model of TBCs with imperfections}

Taking into consideration the relatively low stiffness of TBC [32], the minor effect of TBC on the shakedown behavior is neglected. Therefore, the multilayered TBCs can be simplified as the trilayered systems. The geometrical models of trilayered systems with and without interface imperfections are shown in Fig.2. According to the experimental observation in thermal barrier coatings, the thickness $h_{o}$ of TGO generally changes from $1 \mu \mathrm{m}$ to $8 \mu \mathrm{m}$, and the thickness of bond coat is 
$50 \mu m$ [2]. The geometrical parameters studied in this research are listed in Table 1. Especially, $R / h_{o}=0$ and $H / R=0$ in Table 1 represents that there is no imperfection at the interface of TGO and Bond layer, as shown in Fig.2a.

The simulations are conducted by the commercial code ABAQUS with the subroutine implementation of the upper bound shakedown analysis based on the linear matching method proposed in the above section. Axisymmetric finite element models are established according to the practical applications, and the axis of symmetry is at the center of the model. The surface boundary of the TGO facing the TBC is considered to be free and the Y-direction displacement at the bottom surface is constrained because the substrate is considered to be deep enough to suppress the overall bending. Typical finite element mesh models for different geometries are shown in Fig.3.

(a) Without imperfection

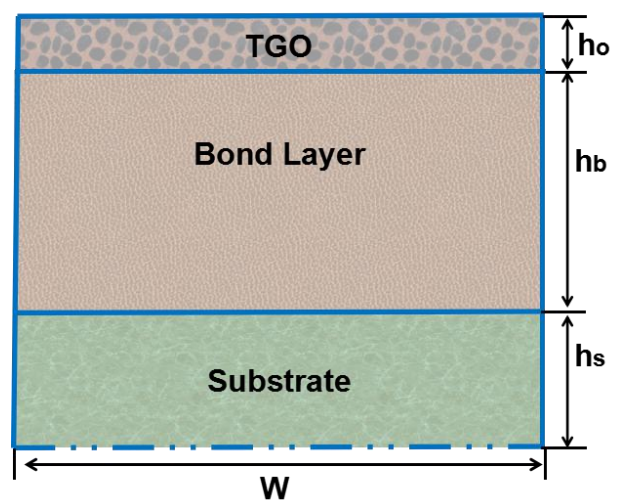

(b) With imperfection

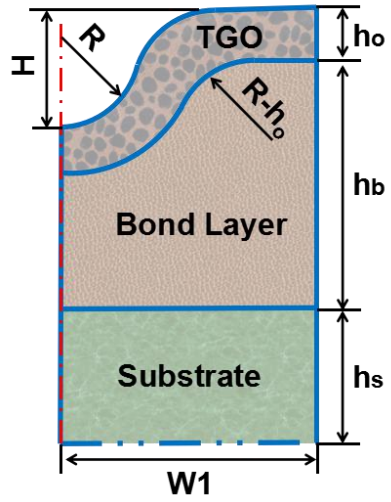

Fig.2 Geometrical models of multilayered systems with and without imperfections

Table 1 Geometrical parameters of multilayered system $(\mu \mathrm{m})$

\begin{tabular}{ccccccc}
\hline$h_{o}$ & $h_{b}$ & $h_{s}$ & $R / h_{o}$ & $H / R$ & $W / h_{o}$ & $W 1 / R$ \\
\hline 1 & 50 & 25 & 0 & 0 & 2 & 5 \\
2 & 50 & 25 & 1.25 & 0.5 & 2 & 5 \\
4 & 50 & 25 & 1.5 & 1 & 2 & 5 \\
6 & 50 & 25 & 1.75 & 1.5 & 2 & 5 \\
8 & 50 & 25 & 2 & 2 & 2 & 5 \\
\hline
\end{tabular}




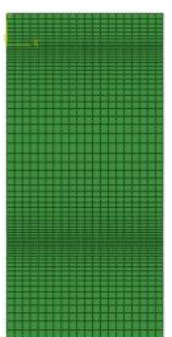

\section{R/ho=0} $H / R=0$

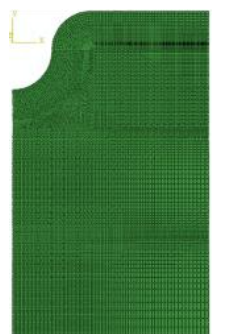

R/ho=1.25 $H / R=2$

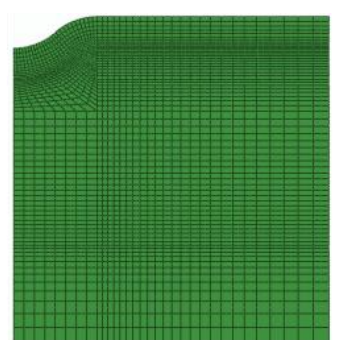

R/ho=2 $\mathrm{H} / \mathrm{R}=0.5$

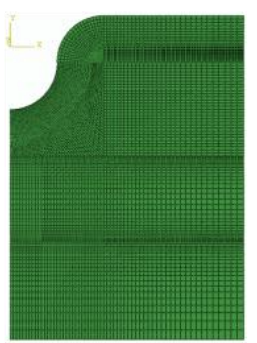

R/ho=1.5 H/R=2

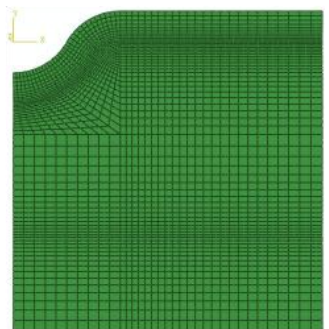

R/ho=2 $H / R=1$

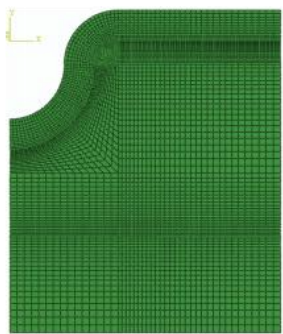

R/ho=1.75 $\mathrm{H} / \mathrm{R}=\mathbf{2}$

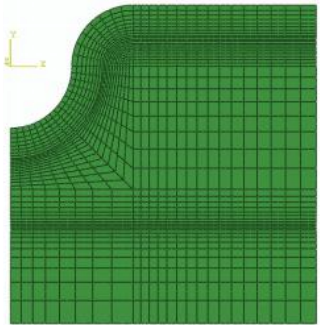

$\mathrm{R} / \mathrm{ho}=\mathbf{2}$

$H / R=1.5$

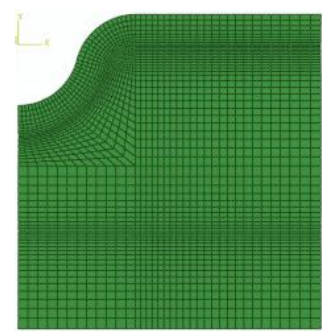

$\mathrm{R} / \mathrm{ho}=2$

$H / R=2$

Fig.3 Finite element models under different geometries for $h o=8 \mu \mathrm{m}$

In this work, the bond coat is assumed to be a elastic-perfectly plastic material with significant temperature dependence. The substrate and TGO are considered to be linear elastic to investigate the cyclic thermal deformation of multilayered TBCs. It should be noted that the impact of the TGO growth on the shakedown behavior is simplified as the thickness effect, and the TGO thickness changes from $1 \mu \mathrm{m}$ to $8 \mu \mathrm{m}$ in this research. The main material parameters of multilayered systems are listed in Table 2.

Table 2 Material parameters of multilayered systems [2, 3]

\begin{tabular}{ccccc}
\hline & $\begin{array}{c}\mathrm{TBC} \\
\end{array}$ & $\mathrm{TGO}$ & Substrate & Bond layer \\
& $\left(\mathrm{ZrO}_{2} / \mathrm{Y}_{2} \mathrm{O}_{3}\right)$ & & & \\
\hline $\begin{array}{c}\text { Young's modulus }(\mathrm{GPa}) \\
\text { Poisson ratio }\end{array}$ & $5-60$ & 380 & 190 & 190 \\
$\begin{array}{c}\text { Thermal expansion } \\
\text { coefficient }\left(\times 10^{-6} /{ }^{\circ} \mathrm{C}\right)\end{array}$ & -- & 0.2 & 0.3 & 0.3 \\
\hline
\end{tabular}


As reported in the literature [2], the yield strength of the bond layer is typically temperature dependent, as shown in Fig.4. Based on the experimental data, the temperature-dependent yield stress can be described by the Boltzmann function, as shown in Eq.(8).

$$
\sigma_{y}^{b c}(T)=A_{1}+A_{2} /\left(1+\exp \left(\left(T-A_{3}\right) / A_{4}\right)\right)
$$

The constants defined in Eq.(1) can be obtained easily by fitting the experimental data. Namely, $A_{1}=57, A_{2}=963, A_{3}=582, A_{4}=81$.

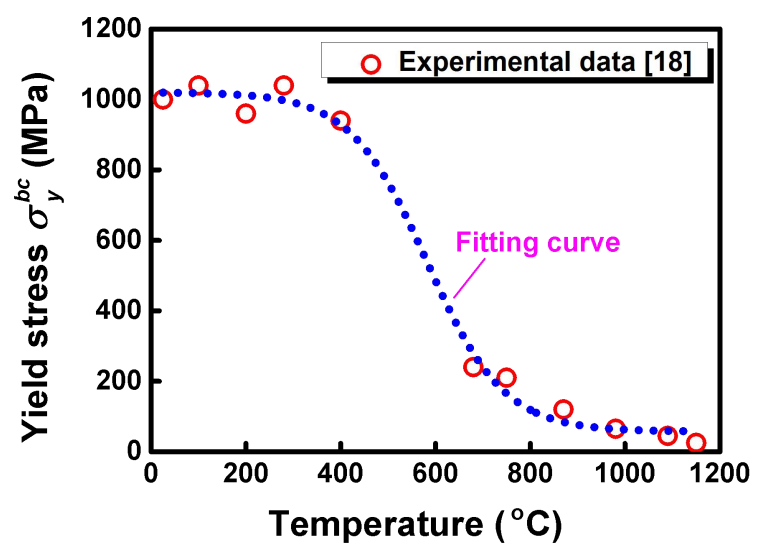

Fig.4 Temperature-dependent yield strength of the bond material

\section{Results and discussions}

When the fixed boundary is applied at the right-hand side of the finite element model, the thermal stresses are produced by constraining the thermal expansion deformation for multilayered TBCs without interface imperfections. In his case, the difference of thermal expansion coefficients has no effect on the thermal stress because there's no relative deformation produced between different layers. Accordingly, the thermal stress for each layer can only be influenced by its thermal expansion coefficient and can be calculated by Eq.(9).

$$
\sigma^{\text {th }}(T)=E \alpha \Delta T /(1-v)
$$


If the interface imperfections are considered, a significant stress concentration phenomenon will take place due to the local geometric discontinuity. The difference between thermal expansion coefficients of different layers shows an important influence near the discontinuity area. Based on the proposed iterative algorithm mentioned above, the convergence processes of shakedown limit load factor $\lambda$ for two cases are described in Fig.5. Results show that the proposed approach has very high efficiency to obtain the shakedown limit loads under different load conditions. It should be noted that the shakedown limit factor $\lambda$ decreases gradually with increasing number of iterations for the temperature-independent material based on the upper bound shakedown theory and the linear matching method. When the temperature-dependent material is used, the shakedown limit factor $\lambda$ with respect to number of iterations may show the numerical fluctuation phenomenon. The reason is that the reduction of the shakedown limit factor $\lambda$ causes the decrease of the applied temperature, but the yield stress significantly increases with reducing the temperature, then the shakedown limit factor $\lambda$ increases during the following iteration step. The numerical fluctuation phenomenon lasts until a reasonable and steady shakedown limit factor $\lambda$ is obtained. The shakedown limits of multilayered TBCs with interface imperfections under different TGO thicknesses are shown in Fig. 6. Noting that the reference temperature $T_{r}$ of this research is defined as $500^{\circ} \mathrm{C}$, and the reference stress is the yield stress corresponding to the reference temperature, namely $\sigma_{r}=761 \mathrm{MPa}$. The calculated shakedown limits are normalized with respect to the reference temperature $T_{r}$ in the longitudinal coordinates, and are divided by the reference stress $\sigma_{r}$ in horizontal ordinates in the following work. Results present that the thickness has no effect on shakedown limits (Fig.6a). This is reasonable because the TGO is considered to be linear elastic and the fixed boundary is assumed in this research. The deformation in the TGO is much less than that produced in the bond layer. Hence, the thickness of the TGO has little effect on the stress state of the bond layer and also the shakedown limits of the TBCs. However, the shape of interface defect shows a remarkable impact on the shakedown limits of structures, as illustrated in Fig. 6b. Based on the simulated data, the impact of geometric parameters at interface 
imperfections on shakedown limits can be formulated in Eq.(10), which can be used to estimate the shakedown limits simply for $R / h_{o}=2$.

$$
T / T_{r}=0.94 \times(1+H / R)^{-0.152}
$$

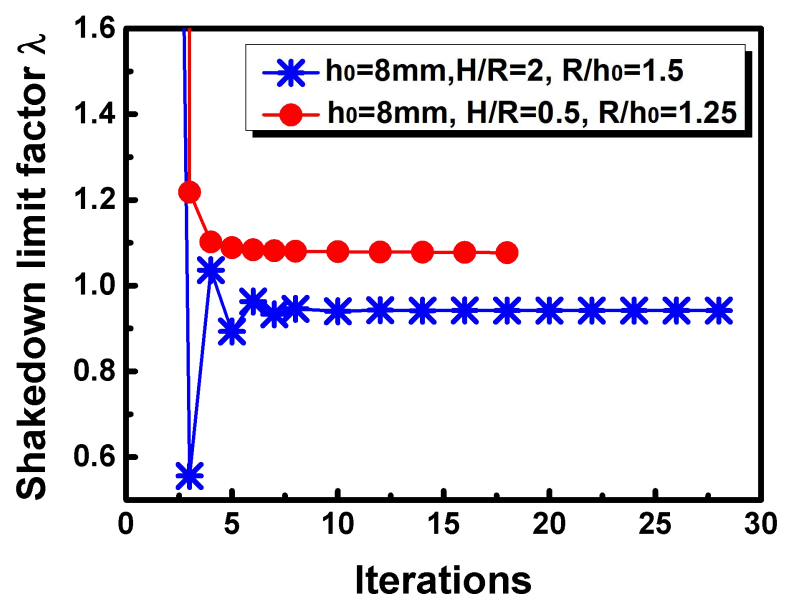

Fig.5 Convergence process of the proposed iterative algorithm for shakedown limits
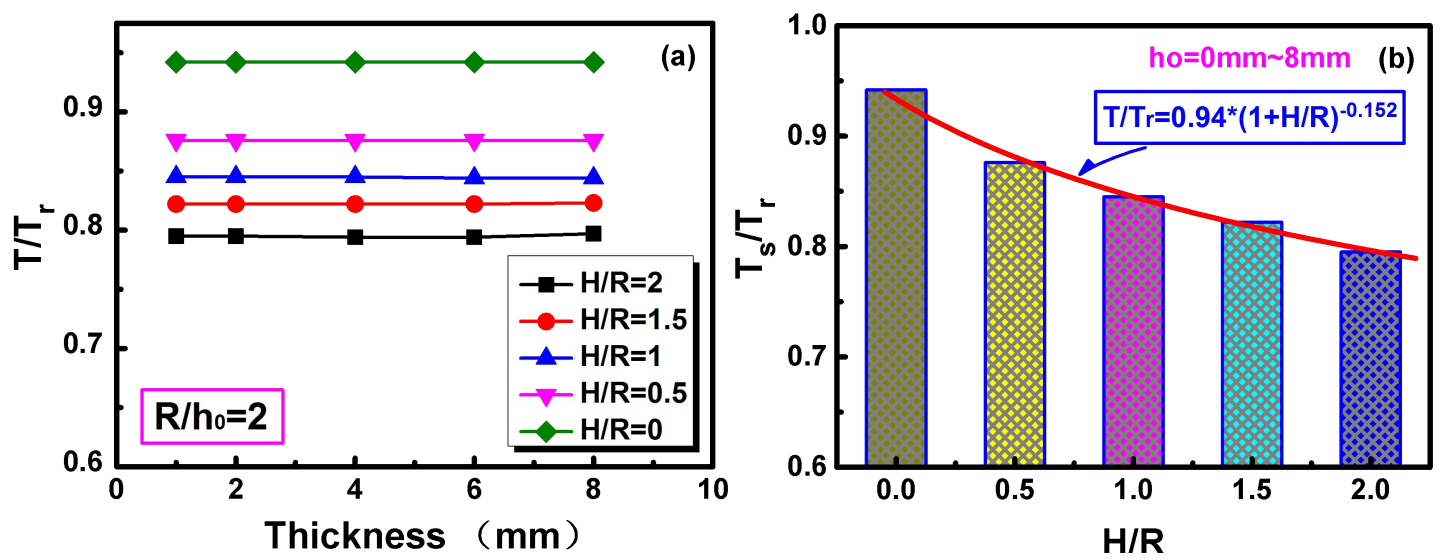

Fig.6 Shakedown limits of multilayered systems under different thicknesses

To investigate the effect of geometric parameters $R / h_{o}$ on the shakedown boundaries, four different geometric parameters $R / h_{o}$ of $1.25,1.5,1.75$ and 2 are further discussed. The thickness of TGO for each case is $8 \mu \mathrm{m}$ and the calculated shakedown limits are illustrated in Fig.7. It should be stressed that the Eq.(11) is independent of thickness of TGO layer, and is appropriate for the shakedown 
assessment of multilayered systems with various interface imperfections since the thickness has no effect on the shakedown limit.

$$
T / T_{r}=0.94 \times(1+H / R)^{-\left(0.096+0.028 R / h_{0}\right)}
$$

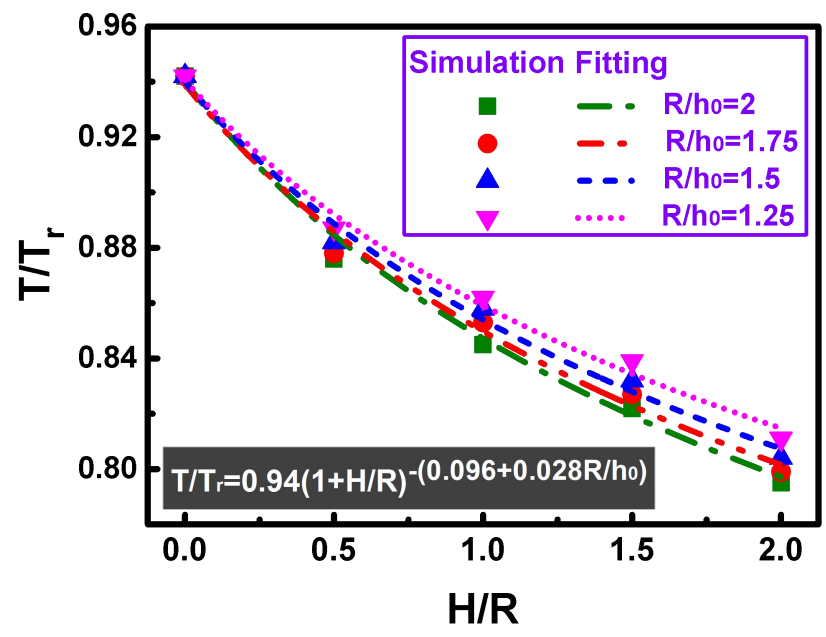

Fig.7 Shakedown limits of multilayered TBCs under different geometries of imperfections

The thermal expansion coefficient of the bond layer may change in a reasonable range due to a slight variation of contents and production technology. Five different thermal expansion coefficient ratios $\alpha / \alpha_{\mathrm{r}}$ of $0.8,0.9,1,1.1$ and 1.2 are researched to analyze the impact of the thermal expansion coefficient of the bond layer on shakedown limits of multilayered TBCs. It is noting that the reference thermal expansion coefficient $\alpha_{\mathrm{r}}$ is defined as $14.3 \times 10^{-6} /{ }^{\circ} \mathrm{C}$ in Table 1 for comparison. The calculated shakedown boundaries for different thermal expansion coefficients and geometric parameters are presented in Fig.8. Results clearly show that the thermal expansion coefficient ratio has a remarkable impact on the shakedown limits for different $R / h_{o}$. It is predictable because the higher thermal stress will occur near the defect region ascribing to the greater thermal mismatch induced by the larger difference of thermal expansion coefficients in different layers. However, the geometric parameter $R / h_{o}$ seems to have a slight influence on the shakedown limits, whereas $H / R$ impacts the shakedown limits greatly under various cases simulated, as 
illustrated in Fig.9. The results are different with the common phenomenon that the transition radius usually has an important action on shakedown limits. This phenomenon can be interpreted by the elastic thermal stress field mainly produced by the geometrical discontinuity for $h_{o}=8 \mu \mathrm{m}$ and $\alpha / \alpha_{\mathrm{r}}=1$ at $300^{\circ} \mathrm{C}$, as shown in Fig. 10 . The maximum von-Mises equivalent stress of the bond layer is $676.5 \mathrm{MPa}, 610.9 \mathrm{MPa}$, 497.2MPa and $382.9 \mathrm{MPa}$ when $R / h_{o}$ equals $2,1.5,1$ and 0.5 for $H / R=2$, but it is 676.5MPa, 660.5MPa, 643.9MPa, and 627.9MPa when $H / R$ equals $2,1.75,1.5$ and 1.25 for $R / h_{o}=2$, respectively. This indicates that the geometrical parameter $R / h_{o}$ obviously has a greater effect on the maximum von-Mises equivalent stress comparing with the geometrical factor $H / R$. Moreover, the position of the maximum von-Mises equivalent stress goes up obviously along the defect boundary when $R / h_{o}$ changes from 2 to 0.5 , but it varies very slightly when $H / R$ decreases from 2 to 1.25 . This indicates the maximum von-Mises equivalent stress locates at the imperfection surface and near the middle-right of the defect boundaries for various geometries models, and the depth of the defect is the main factor for the maximum von-Mises equivalent stress. Actually, the geometrical parameter $R / h_{o}$ influences the defect depth greatly, but $H / R$ mainly changes the transition radius at the upper-right corner of the imperfection. According to the simulated results, the stress near the upper-right corner of the imperfection is significantly smaller comparing with that in the other region. This implies that the transition radius at the upper-right corner of the imperfection has only a little influence on the stress distribution. According the above discussion, the shakedown limit of multilayered TBCs, which is influenced by the maximum von-Mises equivalent stress directly, reduces markedly by increasing the defect depth described by $R / h_{o}$, but changes slightly with varying the transition radius of the imperfection represented by $H / R$. 

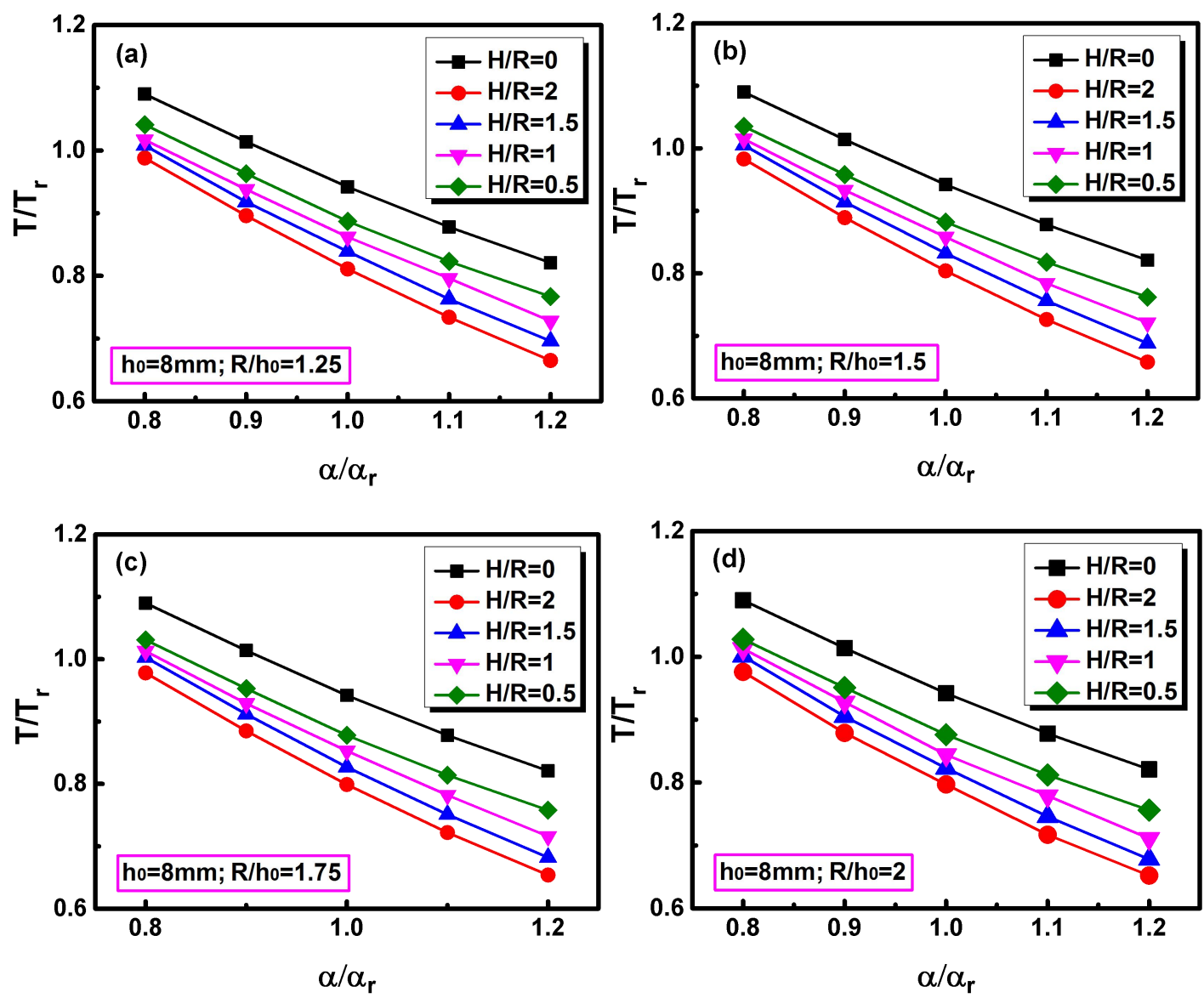

Fig. 8 Effect of the thermal expansion coefficients on the shakedown limits
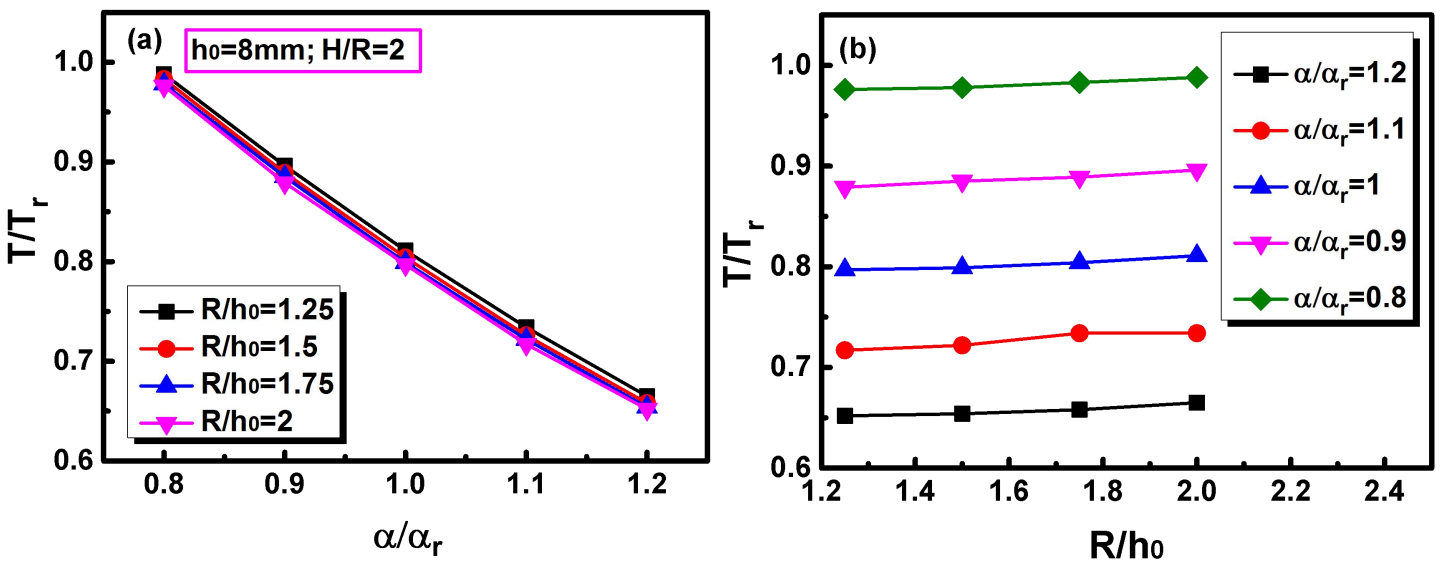

Fig. 9 Effect of the geometric parameter $R / h_{o}$ on the shakedown limits 


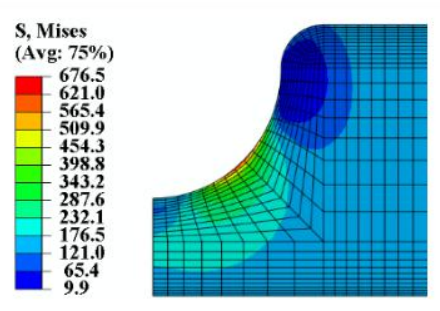

(a) $H / R=2, R / h o=2$

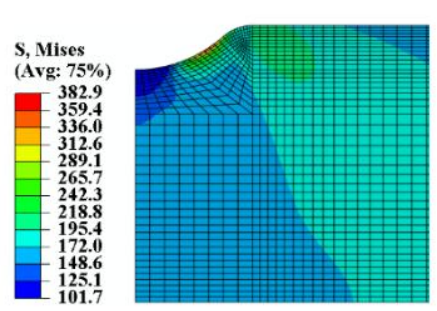

(d) $H / R=2, R / h o=0.5$

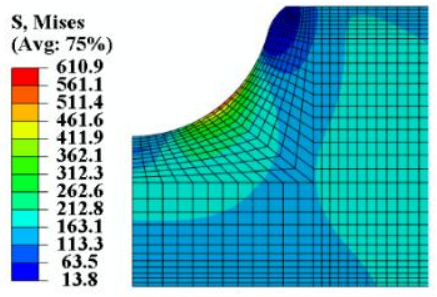

(b) $H / R=2, R / h o=1.5$

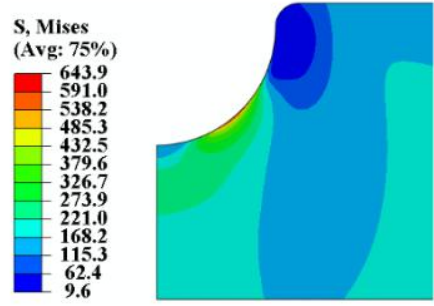

(e) $H / R=1.5, R / h o=1.5$

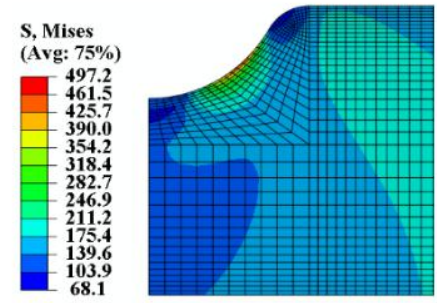

(c) $H / R=2, R / h o=1$

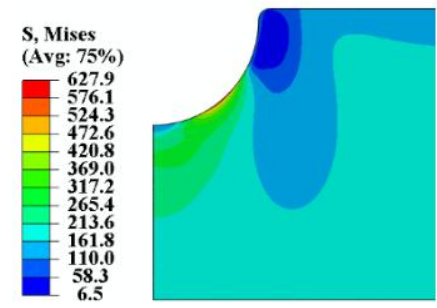

(f) $H / R=1.25, R / h o=1.5$

Fig. 10 Elastic thermal stress field of the bond layer with interface defects for $h_{o}=8 \mu \mathrm{m}$ and $\alpha / \alpha_{\mathrm{r}}=1$ at $300^{\circ} \mathrm{C}$

To assess the shakedown limits of multilayered structures with interface imperfections easily, the shakedown limits are fitted according to the calculated data, as presented in Eq. (12). Since the geometrical parameter $R / h_{o}$ has little effect on the shakedown limits, the parameter $R / h_{o}$ is not considered in Eq. (12) for simplicity. It is worth noting that the more accurate estimation can also be achieved easily similar to Eq. (11), but the Eq. (12) is definitely acceptable for shakedown evaluation in practical engineering owing to the sufficiently small error. It should be noted that the effect of the elastic modulus on shakedown limits is the same as that of the thermal expansion coefficient, which is not further discussed in the following work.

$$
T / T_{r}=(2.46+0.38 H / R) \times\left(1+T_{r} / T\right)^{-(1.41+0.31 H / R)}
$$

Effective plastic strains of the bond layer above shakedown limits for various geometric conditions are illustrated in Fig.11. Results indicate that the effective plastic strain takes place at the interface between the TGO layer and the bond layer. Combining with Fig.1 and Fig.11, it can be clearly concluded that the crack failure at the interface between the TGO layer and the bond layer is mainly caused by the 
accumulated plastic deformation in this area produced by the repeated thermal loads. Similar conclusion has been achieved by Karlsson et al. [2] based on the finite element simulation and observed by Mumm et al. [3] in the microscopic experiment analysis. In this research, the shakedown boundaries are further obtained and discussed, which is very important for safety assessment and engineering design for multilayered TBCs in practical applications.

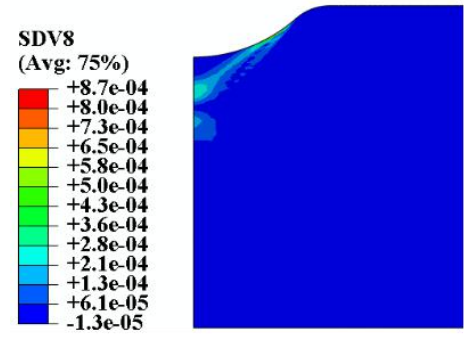

(a) $H / R=2, R / h o=0.5$

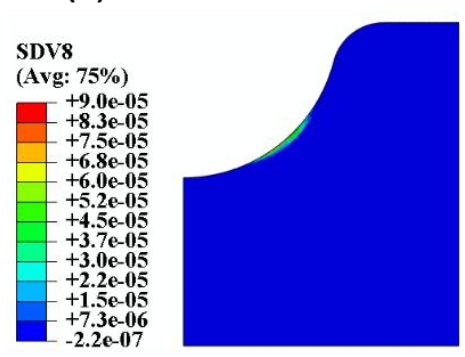

(c) $H / R=2, R / h o=1.5$

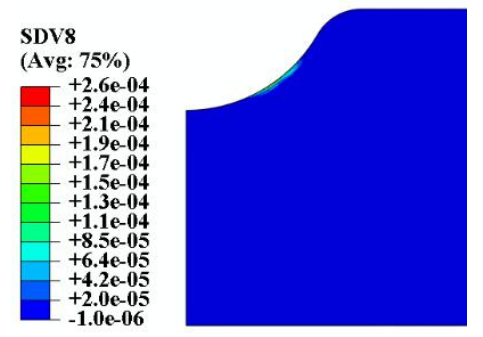

(b) $H / R=2, R / h o=1$

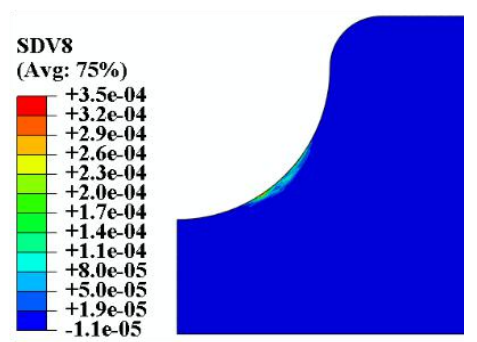

(d) $H / R=2, R / h o=2$

Fig. 11 Effective plastic strains of the bond layer above the shakedown limit

\section{Conclusions}

The shakedown boundaries of multilayerd systems considering the impacts of interface imperfections are investigated according to the proposed linear matching method, in which the temperature-dependent yield stress of the bond layer is considered to simulate the real material performance. Some important influence factors, such as the geometric parameters $h_{o}, R / h_{o}$ and $H / R$ as well as the thermal expansion coefficient ratio $\alpha / \alpha_{\mathrm{r}}$ are discussed systematically. The main conclusions include that the thickness of the TGO has no effect on shakedown limits of the multilayered TBCs due to the assumption of the linear elastic behavior of TGO and 
the fixed boundary. It conforms to the practical application because the elastic modulus of the TGO is much greater than that of the bond layer. However, the geometric parameter $H / R$ have a remarkable effect on the shakedown limits, but the geometric factor $R / h_{o}$ shows a slight influence under various cases. This because the geometrical parameter $R / h_{o}$ influences the defect depth greatly, but $H / R$ mainly changes the transition radius at the upper-right corner of the imperfection. According the elastic thermal stress analysis, the defect depth is the prominent factor for the maximum von-Mises equivalent stress at the interface of the imperfection. Whereas, the transition radius at the upper-right corner of the defect, which usually has an important impact on the local stress distribution of structures, has only a little influence on the maximum von-Mises stress in this cases. Additionally, the thermal expansion coefficient ratio has a remarkable effect on the shakedown boundary ascribing to the thermal mismatch induced by the difference of thermal expansion coefficients. The assessment approach of shakedown limits for multilayered TBCs considering various factors mentioned above is proposed in Eq.(12) based on the obtained data. It is worth noting that the effective plastic strain above the shakedown limit load takes place at the interface between the TGO layer and the bond layer. This phenomenon is very similar to the crack failure behavior achieved by Karlsson et al. [2] according to the finite element simulation and obtained by Mumm et al. [3] based on the microscopic observation, as shown in Fig. 1 and Fig. 11.

\section{Acknowledgements}

The authors gratefully acknowledge the support of the China Scholarship Council (201608420201), the University of Strathclyde and Wuhan Youth Science and technology plan (2016070204010120) during the course of this work.

\section{References}

[1] Padture N. P., Gell M., Jordan E. H., 2002. Thermal barrier coatings for gas-turbine engine applications. Science 296: 280-284. 
[2] Karlsson A. M., Evans A. G., 2001. A numerical model for the cyclic instability of thermally grown oxides in thermal barrier systems. Acta Mater. 49: 1793-1804.

[3] Mumm D. R., Evans A. G., Spitsberg I. T., 2001. Characterization of a cyclic displacement instability for a thermally grown oxide in a thermal barrier system. Acta Mater. 49: 2329-2340.

[4] Hsueh C. H., 2002. Modeling of elastic deformation of multilayers due to residual stresses and external bending, J. Appl. Phys. 91: 9652-965.

[5] Zhang N. H., 2007. Thermoelastic stresses in multilayered beams. Thin Solid Films 515: 8402-8406.

[6] Hu Y. Y., Huang W. M., 2004. Elastic and elastic-plastic analysis of multilayer thin films: Closed-form solutions. J. Appl. Phys. 96: 4154-4160.

[7] Zhang X. C., Xu B. S., Xuan F. Z., 2008. Residual stresses in the elastoplastic multilayer thin film structures: The cases of $\mathrm{Si} / \mathrm{Al}$ bilayer and $\mathrm{Si} / \mathrm{Al} / \mathrm{SiO} 2$ trilayer structures, J. Appl. Phys. 103: 073505.

[8] Sadigh M. A. S., 2016. Creep simulation of adhesively bonded joints using modified generalized time hardening model, J. Mech. Sci. Technol. 30: 1555-1561.

[9] Chen Q. Q., Xuan F. Z. and Tu S. T., 2009. Residual stress analysis in the film/substrate system with the effect of creep deformation, J. Appl. Phys. 106: 033512.

[10]Chen Q. Q., Xuan F. Z. and Tu S. T., 2009. Modeling of creep deformation and its effect on stress distribution in multilayer systems under residual stress and external bending. Thin Solid Films 517: 2924-2929.

[11]Limarga A. M, Wilkinson D. S., 2006. A model for the effect of creep deformation and intrinsic growth stress on oxide/nitride scale growth rates with application to the nitridation of $\lambda$-TiAl. Mater. Sci. Eng. A. 415: 94-103.

[12]Mao W. G., Zhou Y. C., Yang L., Yu X. H., 2006. Modeling of residual stresses variation with thermal cycling in thermal barrier coatings, Mech. Mater. 38: 1118-1127.

[13]Mahbadi H., Gowhari A. R., Eslami M.R., 2004. Elastic-plastic-creep cyclic loading of beams using the Prager kinematic hardening model, J. Strain. Anal. Eng. 39: 127-136.

[14]Nakane K., Ohno N., Tsuda M., Yagi Y. J., Nakagawa I., Atsumi T., 2008. Thermal ratcheting of solder-bonded elastic and elastoplastic layers. Int. J. Plasticity 
24: 1819-1836.

[15]Nakane K., Ohno N. and Tanie H., 2009. Thermal ratcheting of solder-bonded layered plates: cyclic recovery and growth of deflection. Comput. Mech., 46 (2) (2009) 259-268.

[16] Zheng X. T., Wang J. Q., Wang W, Ma L. W., Lin W., Yu J. Y., 2018. Elastic-plastic-creep response of multilayered systems under cyclic thermo-mechanical loadings. J. Mech. Sci. Technol. 32: 1227-1234.

[17] Zheng X. T., Xuan F. Z., 2012. Shakedown analysis of multilayered beams coupled with ductile damage. Nucl. Eng. Des. 250: 14-22.

[18] Zheng X. T., Peng C. F., Yu J. Y., Wang, C. G., Lin, W., 2015. A unified shakedown assessment method for butt welded joints with various weld groove shapes. ASME J. Pres. Ves. Technol., 2015, 137(2): 021404.

[19] BSI, 2014, “ Unfired Pressure Vessels_-Part 3: Design,” BSI Standards, Brussels, Belgium, Standard No. EN 13445-3.

[20] Nguyen-Tajan T. M. L, Pommier B., Maitournam H., et al. 2003, Determination of the stabilized response of a structure undergoing cyclic thermal-mechanical loads by a direct cyclic method. In: ABAQUS users' conference proceedings.

[21] Chen, H., Ponter, A.R., 2001. A method for the evaluation of a ratchet limit and the amplitude of plastic strain for bodies subjected to cyclic loading. Eur. J. Mech.-A/Solids 20, 555-571.

[22] Chen, H., Ponter, A.R., 2010. A direct method on the evaluation of ratchet limit. ASME J. Pres. Ves. Technol. 132: 041202.

[23] Adibi-asl, R., Reinhardt, W., 2011, Non-cyclic shakedown/ratcheting boundary determination - Part 2: Numerical implementation. Int. J. Pres. Ves. Pip. 88: 321-329.

[24] Zheng X., Peng H., Yu J., and Wang W., 2017. Analytical Ratchet Limit for Pressurized Pipeline under Cyclic Nonproportional Loadings. J. Pipeline Syst. Eng. Pract. 8: 04017002.

[25] Mackenzie, D., Boyle, J. T., and Hamilton, R., 2000. The Elastic Compensation Method for Limit and Shakedown Analysis: A Review. J. Strain Anal. Eng. Des. 35: 171-188.

[26]Seshadri, R., 1995. Inelastic Evaluation of Mechanical and Structural Components Using the Generalized Local Stress Strain Method of Analysis. Nucl. Eng. Des. 153: 287-303. 
[27] Vu, D. K., Yan, A. M., and Nguyen-Dang, H., 2004. A Primal-Dual Algorithm for Shakedown Analysis of Structures. Comput. Methods Appl. Mech. Eng. 193: 4663-4674.

[28] Staat, M., and Heitzer, M., 2001. LISA a European Project for FEM-based Limit and Shakedown Analysis. Nucl. Eng. Des. 206: 151-166.

[29] Koiter, W. T., 1960. General Theorems for Elastic Plastic Solids. Progress in Solid Mechanics 1, Sneddon J. N. and Hill R., eds., North-Holland, Amsterdam, $167-221$.

[30] Chen, H. F., and Ponter, A. R. S., 2001. Shakedown and Limit Analyses for 3-D Structures Using the Linear Matching Method. Int. J. Pres. Ves. Pip. 78: 443-451.

[31] Hibbitt, K., 1997. ABAQUS: User's Manual. Hibbitt, Karlsson \& Sorensen, Incorporated.

[32] Mumm D. R., Watanabe M., Evans A. G., Pfaendtner J.A., 2004, The influence of test method on failure mechanisms and durability of a thermal barrier system. Acta Mater. 52: 1123-1131. 